\title{
3 Electrophysiological Localization
}

In this chapter, we describe our technique of electrophysiological mapping of M1 and the CS (Arle and Shils 2011, Shils and Arle 2011, Shils and Deletis 2013). The surgical entry point is determined by moving approximately $1-2 \mathrm{~cm}$ behind the midpoint between the nasion to inion toward the lateral canthus or anterior margin of the tragus using a curved scalp incision. This allows for a craniotomy -if elected- that measures roughly 5-6 cm in diameter and gives enough access for small adjustments either slightly superiorly or inferiorly along the convexity to map face or hand as desired. We do not use a Mayfield head holder since the head does not need to be rigidly held in place and if the patient has a seizure induced by mapping further injury can be avoided by not having them pinned in a rigid head-holder.

\subsection{Somatosensory Mapping}

This consists of placing the lead on the dura in a variety of directions, mostly perpendicular to the suspected pre-central gyrus. Median and/or ulnar nerve somatosensory evoked potential's (SSEP's) are then run using a $20 \mathrm{~mA} 200 \mu \mathrm{s}$ monopolar square pulse at a rate of $4.32 \mathrm{~Hz}$. SSEP stimulating electrodes are placed at the wrist overlying either the median or ulnar nerves with the anode placed distally to that cathode. SSEP's are recorded from the lead in both bipolar (contact $1-2,2-3, \ldots$ ) and monopolar (all referenced to the 10-20 location of $\mathrm{Fz}$ ) recording montages. Otherwise, the median nerve is stimulated bipolarly at a frequency of 2-3 Hz and an intensity of 5-20 mA until slight twitches of the thumb are obtained. A ground electrode is placed around the forearm with a loop tape strap. Recordings are performed by using a filter band pass of $20-2000 \mathrm{~Hz}$, a time base of $50 \mathrm{~ms}$, and a grid of multiple contacts (12-24) or the epidural lead that is intended to be implanted and used for chronic stimulation. The main cortical components of the median nerve SSEPs include a positive response (downward peak) over the precentral (motor) gyrus with a mean latency of 20 milliseconds (P20) and a negative response (upward peak) over the postcentral (sensory) gyrus with a mean latency of 20 milliseconds (N20). The CS is determined as the point where the N20 response phase reverses (Fig.3.1) In order to get a complete picture of the CS, multiple mapping locations are needed.

This technique has several limitations. First, cortical SSEPs are absent in the case of severe or complete sensory deafferentation (e.g. amputation). Second, SEP phase reversal is not reliable for the stimulation of the face (trigeminal nerve) or the lower limb (tibial nerve). Third, the P20 component can be missing, even in awake or mildly sedated patients. Fourth, tortuosity of the CS, that in some parts becomes parallel 
to the midsagittal line, complicates the placement of the 4-contact strip on M1 by determining reversal only in a single point. Fifth, there may be a $1-2 \mathrm{~cm}$ discrepancy between SSEP maximum in SI and the hand motor area in M1, making SSEP targeting inaccurate (see Saitoh and Hosomi 2009 and Pirotte et al 2009 for full discussion). In practice, SSEP have been sidestepped by several authors.

According to Velasco et al (2002), recording corticocortical evoked responses (CCER) is simple, reliable, and superior to SSEPs. MI stimulation elicits negative CCER over the frontal scalp, whereas SI stimulation elicits positive responses over parietal and occipital scalp regions.

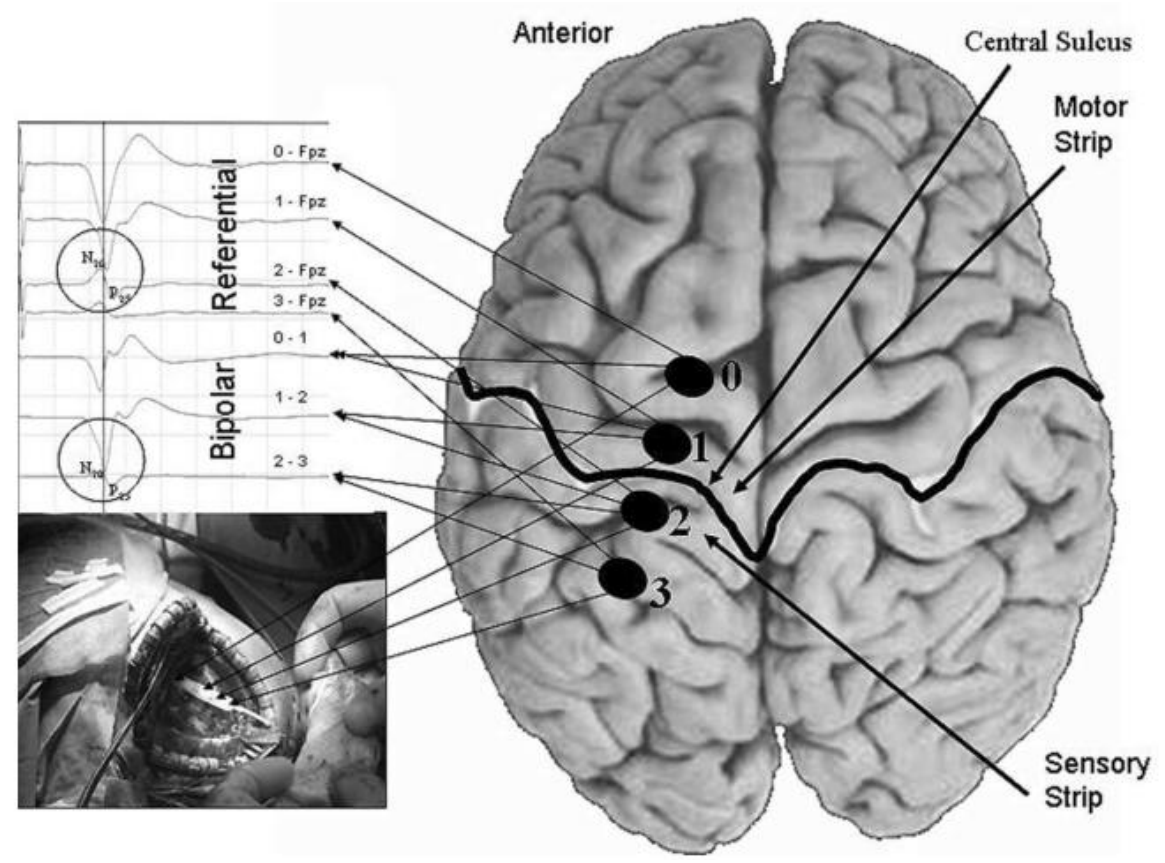

Fig. 3.1: Use of the SSEP reversal potential method to determine location of M1 extradurally. Contacts 0-3 of a typical 4-contact paddle-type electrode are shown in one example placement across the underlying central sulcus. The upper left waveforms show the SSEP in each contact. The reversal of phase occurs, in this example, between contacts 1 and 2 . The inset picture at lower left is the intraoperative photo of this technique being used, revealing the relative size of the lead and the craniotomy opening. By moving the lead around in different locations, the path of the sulcus can be mapped out on the dura. 


\subsection{Motor Mapping}

This is the most crucial step for optimizing M1 ICS electrode placement, i.e. performing intraoperative motor mapping (MM), which consists of activating M1 via electrical stimulation and recording the responses in specific muscle groups (Shils and Deletis 2011, Azabou et al 2013). Activation of M1 causes action potentials to travel down the corticospinal tract to alpha-motor neurons $(\alpha M N)$ in the ventral spinal cord. Once an $\alpha \mathrm{MN}$ is activated actions potentials travel to muscle and generate a compound muscle action potential (CMAP). It goes without saying that MM cannot be exploited in patients with total or severe motor deficits or with missing limbs.

There are 3 important factors in mapping motor regions: (1) the strength of the stimulus; (2) the type of anesthesia; (3) and the type of stimulator probes used. First, when stimulating M1, the spread of the electric field will determine the resolution of the response. If the stimulus is too low, too few axons will be activated to generate a recordable CMAP. If the stimulus is too high, axons that are more distant (a few mm to $1 \mathrm{~cm}$ ) may be activated and potentially falsely identify a region as M1. Additionally if there is excessive fluid in the surgical field the stimulus will be spread over a larger area, and therefore it is important to maintain a dry area for stimulation. Second, the $\alpha M N$ is very susceptible to the effects of anesthesia. The $\alpha \mathrm{MN}$ is a highly modulated cell with many inputs that can adjust the firing threshold of the cell. When the patient is under anesthesia, the $\alpha$ MN's membrane is more inhibited due to the lack of basal background activity from its multiple inputs. Under such conditions it becomes difficult for a single axon to cause the $\alpha \mathrm{MN}$ to fire. Different anesthetics have varying amounts of inhibitory effects on the $\alpha \mathrm{MN}$ membrane. Thus it is highly recommended to use a total-intravenous anesthetic (TIVA) for these procedures (Sloan 1998), i.e. continuous dose of propofol $(75 \mathrm{mcg} / \mathrm{kg} / \mathrm{min}$ up to $150 \mathrm{mcg} / \mathrm{kg} / \mathrm{min}$ ) combined with either fentanyl or remifentanil $(0.05 \mathrm{mcg} / \mathrm{kg} / \mathrm{min}$ up to $0.5 \mathrm{mcg} / \mathrm{kg} / \mathrm{min}$ ) and no nitrous inhalational agent or muscle relaxants. Yet, even with TIVA, the membrane needs the help of temporal summation at the corticospinal axon input to counteract the relative loss of spatial summation. Historically this has been solved by using a low frequency (50 to $60 \mathrm{~Hz}$ ) long train (1 second or more) of stimuli. This technique, however, can incur up to $11 \%$ seizure rates (Yingling et al 1999). A second technique, known as high-frequency, involves using a train of 5-7 stimuli with a 2-4 ms inter stimulus interval (ISI) (Taniguchi et al 1993). This technique has demonstrated a much lower seizure frequency rate of 1.6\% (Szeleny et al 2006) and significantly increases the 'on display' signal-to-noise ratio. Both bipolar stimulation (the anode and cathode are within $1 \mathrm{~cm}$ of each other) and monopolar stimulation (the cathode is placed at some far reference point on the body, usually the contralateral 10-20 system F1 or F2 position) can be employed. Although bipolar stimulation is supposed to offer a more focal stimulation response, if the field is kept dry and the stimulation is adjusted to the threshold response, monopolar stimulation fields remain focal. Furthermore, the location of the stimulus is directly under the electrode rather than at some arbitrary point between the two electrodes. 
To record CMAP activity from the muscles, pairs of needles are placed in bipolar fashion (separated by $0.5-2 \mathrm{~cm}$ ) in the orbicularis oculi, orbicularis oris, trapezius, deltoid, biceps, triceps, flexor carpi ulnaris, abductor pollicis brevis, first dorsal interosseous, quadriceps, anterior tibialis, and abductor hallucis muscles (MacDonald et al 2013). Surface electrodes can also be used, but we have found that they can easily be dislodged during the procedure and are more affected by noise. Stimulation consists of trains of 5-9 stimuli, each with an ISI of 250-500 ms, and a $500 \mu \mathrm{s}$ pulse width. Once the probe is placed on the dura stimulation amplitudes are slowly increased starting at $5 \mathrm{~mA}$ (for subdural testing the amplitude is started at $3 \mathrm{~mA}$, though placement in the subdural space is rarely used) and increased to a maximum of $25 \mathrm{~mA}$ or until a response is found. Stimulation can be performed with any EMG system that allows for short low amplitude high frequency trains. When a CMAP response is generated (Fig.3.2), the location is marked on the dura with a sterile pen. Multiple areas are tested to map M1 to provide for optimal electrode placement. It is useful to have ice cold saline kept on the field with which to irrigate, should a seizure occur, as well as having lorazepam available in the OR. EEG can be monitored both during stimulation in order to identify seizure activity, as well as, more importantly, after discharge activity which is an indicator of potential seizure activity, allowing for a more timely cessation of stimulation in that area of the brain.

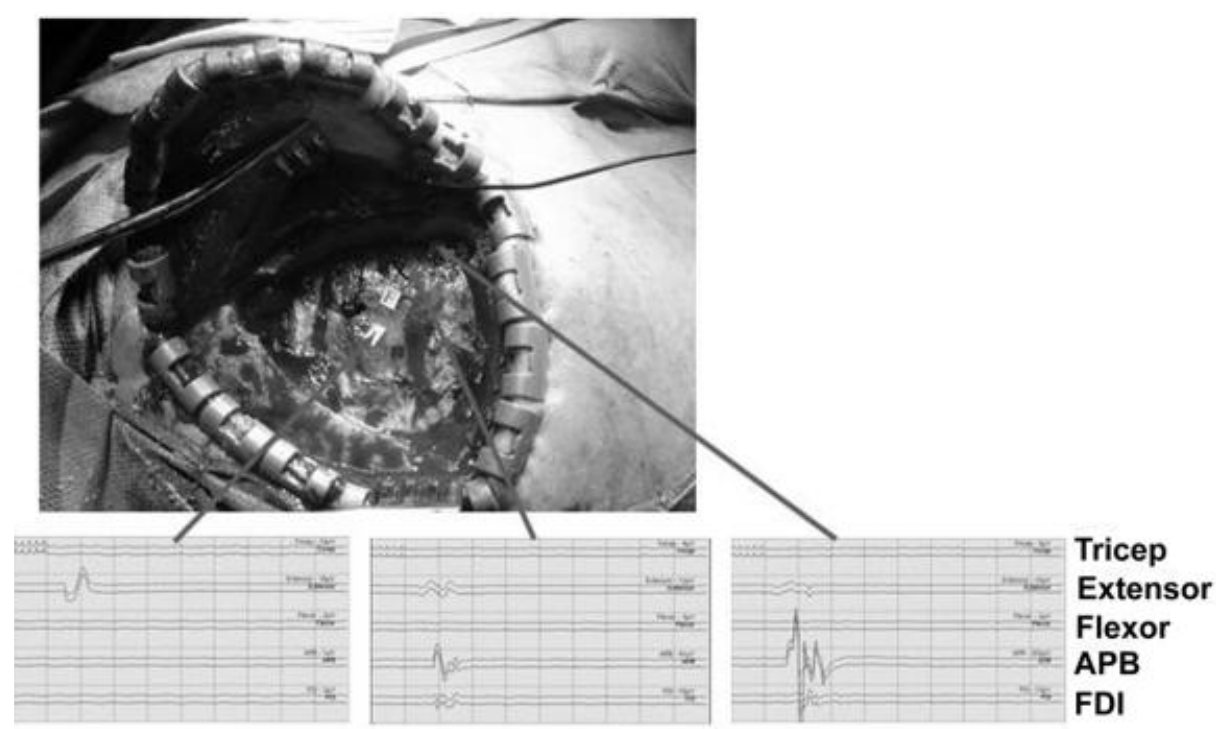

Fig. 3.2: Use of the cortical mapping technique for determing location of M1 regions. The EMG from muscles in the upper extremity are shown below following stimulation with a ball tip electrode in three locations on the dura. The far left shows activation of the extensor muscle in the forearm, the other two show activation of the APB muscle in the hand. This technique corroborates the SSEP method in figure 1 and helps determine more precisely the underlying thresholds for individual muscle groups. 
Other protocols exist in the literature. Teixeira et al (2013) carry out transdural functional cortical mapping with the patient fully awake under local anesthesia. Transdural electrical stimulation exploit the following parameters: 4-6 mA, 30-60Hz, PW 1 ms. Lefaucheur and DeAndrade (2009) perform monopolar anodal stimulation (single square waves of $1 \mathrm{~ms}$ duration, delivered at an intensity range of 10-50 mA), each contact of the epidural grid or quadripolar lead being successively activated as the anode. The cathode (reference electrode) is a subcutaneous needle or a steel plate electrode placed in the occipital region, to avoid direct activation of face muscles by the stimulation; the ground electrode is placed around the forearm with a loop tape strap. EMG recordings detect motor responses at lower intensity thresholds than visual inspection. The MEPs are recorded using a filter band pass of $20-2000 \mathrm{~Hz}$, a time base of 50-100 ms, and surface or subcutaneous needle electrodes placed over the target muscles. The infusion of anesthetic agents (propofol and remifentanil) is carefully monitored to maintain the bispectral index (BIS) around a value of 60 . In rare cases, a single train of two or three stimuli ( $20 \mu$ s interpulse interval) may be required. They recorded intraoperative MEPs in any part of the body. The motor threshold is determined for evocation of a reproducible MEP of more than $100 \mathrm{mV}$ in amplitude in the target territory. For this purpose, the contact of the epidural grid or quadripolar lead that theoretically corresponds to the optimal motor cortical representation of the painful region (according to image-guided navigation and intraoperative SEP mapping) is selected as the anode. With the use of this montage, stimulation intensity is gradually increased by increments of $2 \mathrm{~mA}$ to a maximum of $50 \mathrm{~mA}$. Cortical mapping is then performed at a fixed intensity set at $20-30 \%$ above the motor threshold. The complete procedure includes MEP recordings to monopolar cortical stimulation, by using successively each contact of the epidural grid or quadripolar lead as an anode. From these recordings, the "best anode" is determined, i.e. the contact providing MEPs of maximal amplitude in the target territory when selected as an anode. Recordings using each contact as a cathode, with the occipital reference as the anode, are possible, but, in their experience, of little or no value. Recording MEPs in the target territory in response to bipolar cortical stimulation, using the "best anode" as the anode and successively each adjacent contact of the grid or quadripolar lead as the cathode, may be useful: the amplitude of the MEPs evoked by the various bipolar combinations can be compared with that produced by the optimal anodal stimulation to estimate how much the contacts adjacent to the "best anode" interfere with this electrode. 


\section{References}

Arle JE and JL Shils (eds) Essential Neuromodulation. New York : Academic Press, 2011

Azabou E, Manel V, Andre-Obadia N, et al. Optimal parameters of transcranial electrical stimulation for intraoperative monitoring of motor evoked potentials of the tibialis anterior muscle during pediatric scoliosis surgery. Neurophysiol Clin. 2013; 43: 243-250

Henderson JM, Heit G, Fisher RS. Recurrent seizures related to motor cortex stimulator programming. Neuromodulation. 2010;13;37-43

Lefaucheur JP, de Andrade DC. Intraoperative neurophysiologic mapping of the central cortical region for epidural electrode placement in the treatment of neuropathic pain by motor cortex stimulation. Brain Stimul. 2009;2:138-48.

MacDonald DB, Skinner S, Shils J, et al. Intraoperative motor evoked potential monitoring - A position statement by the American Society of Neurophysiological Monitoring. Clinical Neurophysiology. 2013;124:2291-2316.

Pirotte BJM, Voordecker P, Levivier M, et al. Principles of surgical implantation and complication avoidance. In. Canavero S (ed) Textbook of therapeutic cortical stimulation. New York: Nova Science, 2009. pp 33-44

Saitoh Y, Hosomi K. From localization to surgical implantation. In: Canavero S (ed) Textbook of therapeutic cortical stimulation. New York: Nova Science, 2009. pp 17-32

Shils JL and Arle JE. Treatment Applications of Cortical Stimulation. In: Winn HR (ed) Youman's Neurological Surgery New York: Elsevier, NY, 2011, $6^{\text {th }}$ ed.

Shils JL and Deletis V. Motor Evoked Potentials. In: Kaye AD and Davis SF (eds) Principles of neurophysiological assessment, mapping, and monitoring. New York: Springer, 2013.

Sloan T. Anesthetic effects on electrophysiologic recordings. J Clin Neurophysiol. 1998:15:217.

Szelényi A, Joksimovic B, Seifert V. Intraoperative risk of seizures associated with transient direct cortical stimulation in patients with symptomatic epilepsy. J Clin Neurophysiol. 2006;23:1-5.

Taniguchi M, Cedzich C, Schramm J. Modification of cortical stimulation for motor evoked potentials under general anesthesia: technical description.Neurosurgery 1993;32:219-26.

Teixeira MJ, Ciampi de Andrade D, Fonoff ET. Intra-operative transdural electric stimulation in awake patient: target refining for motor cortex stimulation. Acta Neurochir Suppl. 2013;117:73-8.

Velasco M, Velasco F, Brito F, et al. Motor cortex stimulation in the treatment of deafferentation pain. I. Localization of the motor cortex. Stereotact Funct Neurosurg. 2002;79:146-67.

Yingling CD, Ojemann S, Dodson B, et al. Identification of motor pathways during tumor surgery facilitated by multichannel electromyographic recording. J Neurosurg. 1999;91:922-927. 